\title{
REPRODUTIBILIDADE INTRA E INTEROBSERVADORES NO ÂNGULO DE COBB EM INDIVÍDUOS ESCOLIÓTICOS
}

\section{INTRA-AND INTEROBSERVER REPRODUCIBILITY IN THE COBB ANGLE IN SCOLIOSIS PATIENTS}

\author{
Fabiana Flores Sperandio ${ }^{1}$ \\ Graziela Morgana Silva Tavares ${ }^{2}$ \\ Gilmar Moraes Santos ${ }^{3}$
}

Sperandio FF; Tavares GMS; Santos GM. Reprodutibilidade intra e interobservadores no ângulo de Cobb em indivíduos escolióticos. Rev. Bras. Cresc. e Desenv. Hum. 2011; 21(1): 21-29.

\section{Resumo:}

o objetivo deste estudo é analisar a variabilidade intra e interobservador do método de Cobb em indivíduos escolióticos. Foram avaliadas trinta e quatro radiografias de sujeitos com escoliose para mensuração do ângulo de Cobb. A avaliação intraobservador foi realizada por um avaliador, enquanto a avaliação interobservador foi realizada por três avaliadores. Foram controlados possíveis erros intrínsecos e extrínsecos de mensuração, mas não houve a pré-seleção das vértebras que caracterizam a escoliose. A concordância intra e interobservador foi avaliada pelo coeficiente de correlação de Pearson ( $\mathrm{p} \leq 0,05)$. Não houve diferenças significativas de julgamento na condição intra e interobservadores. A magnitude dos coeficientes de correlação oscilou de bom até excelente nos níveis torácico e tóraco-lombar. Na coluna lombar não houve correlação em ambas as condições.

Palavras-chave: escoliose; ângulo de Cobb; reprodutibilidade inter e intra-observador.

1 Doutora em Engenharia de Produção e Sistemas - UFSC; Professora do Departamento de Fisioterapia da Universidade do Estado de Santa Catarina (UDESC), Rua Pascoal Simone, 358 - Bairro Coqueiros - Florianópolis - Santa Catarina - Brasil CEP: 88080-350.

2 Mestre em Ciências do Movimento Humano - UDESC, Professora do Departamento de Fisioterapia da Universidade Federal do Pampa (UNIPAMPA) BR 472 - Km 585 - Caixa Postal 118 - Uruguaiana - RS - Brasil - CEP: 97500-220.

3 Doutor em Fisioterapia (Ph. D.), Professor do Departamento de Fisioterapia da Universidade do Estado de Santa Catarina (UDESC) Rua Pascoal Simone, 358 - Bairro Coqueiros - Florianópolis - Santa Catarina - Brasil - CEP: 88080-350. Instituição: Universidade do Estado de Santa Catarina - UDESC - Centro de Educação Física, Fisioterapia e Desportos CEFID. Este estudo é parte da dissertação de mestrado, defendida no ano 2000, do programa de pós-graduação em Ciência do Movimento Humano da UDESC/CEFID.

Correspondencia para: Dra. Fabiana Flores Sperandio. Rua: Almirante Lamego, 910, Bl. B, apto: 804 - Centro/Florianópolis/ SC. CEP: 88015-601 - E-mail: d2fs@udesc.br 


\begin{abstract}
:
the aim of this study is to assess intra and interobserver variability of the Cobb method in subjects who have scoliosis. Thirty four scoliosis radiographs were evaluated for measure of the angle of Cobb. The intra-observer assessment was carried out by an observer, while the inter-observer evaluation was carried out by three observers. It was controlled intrinsic and extrinsic errors of measurement, but there was no pre-selection of the vertebrae that characterized the scoliosis. Both intraobserver and interobserver reliabilities were assessed by calculating the Pearson correlation coefficient (p d"0.05). No significant differences were observed between intra-and interobservers. The magnitude of correlation coefficients ranged from very high to unreasonable in chest and thoracolumbar level. Lumbar spine did not correlate in both conditions.
\end{abstract}

Key words: scoliosis; Cobb’s angle; Inter and intra-observer reliability.

\section{INTRODUÇÃO}

A assimetria de tronco é um fenômeno comum durante o crescimento puberal em meninos e meninas ${ }^{1}$. A escoliose, ou curvatura lateral da coluna, pode ser de origem idiopática, congênita ou neuromuscular. A escoliose idiopática é a responsável por $85 \%$ a $95 \%$ de todos os casos de escoliose e sua ocorrência, é maior em mulheres ${ }^{1,2}$.

A identificação da gravidade da deformidade, o planejamento terapêutico subsequente e o prognóstico dessa alteração escoliótica ${ }^{1,3}$ ratificam a necessidade de um exame radiográfico preliminar $^{1,4}$. Assim, novos e sucessivos exames, na busca de melhor qualidade nas imagens e acurácia nas interpretações classificatórias, acabam expondo o indivíduo a altas doses de radiações (próximo a 10.8 cGy) e as consequências do acúmulo de radiação, podem aparecer com o tempo, tais como: leucemia, câncer de mama e defeitos hereditários ${ }^{5-8}$.

A classificação da escoliose é obtida por meio do ângulo de Cobb, método preconizado pela Scoliosis Research Society of North América. Embora seja considerado método "padrão-ouro”, as curvas são avaliadas mediante projeções em filmes radiográficos planos, podendo em muitas situações, avaliar apenas uma das faces das curvaturas tridimensionais da escoliose $^{2,9,10}$.
Mesmo com a padronização metodológica do ângulo de Cobb, ainda assim a literatura aponta discrepâncias e divergências quanto aos valores inter e intra-observador ${ }^{11,12,13}$. Especificamente, as variações interobservadores foram de $2,3^{\circ}$ a $7,2^{\circ}{ }^{13-17}$ e de intra-observador de $2^{\circ}$ a 4,9 ${ }^{\circ 2-16}$ nos valores do ângulo de Cobb.

Sabe-se que mensurações padronizadas do ângulo de Cobb, vinculadas ao controle de possíveis fontes de erro (intrínseco e extrínseco) de medida, proporcionariam maior segurança quanto à efetividade do diagnóstico e, consequentemente, à conduta terapêutica ${ }^{18}$. Os problemas nas mensurações foram atribuídos às constantes trocas de instrumentos durante as mensurações e a não pré-seleção das vértebras. Não houve correlação com a experiência prévia dos observadores. Por outro lado, as principais fontes de erro extrínseco mantiveram relação com a posição do paciente, a posição e distância do tubo durante o exame de raiox e com a hora na realização do exame ${ }^{13,18}$.

Neste estudo sobre a reprodutibilidade do padrão-ouro "ângulo de Cobb", controlouse o erro intrínseco e extrínseco de media, conforme os critérios propostos por Morrissy et $a l^{13}$ e Modi et $a l^{18}$ à exceção da pré-seleção vertebral, por entender-se ser este, um procedimento distante da clínica diária de ortopedistas, cirurgiões ortopédicos e dos demais profissionais. Mesmo assim, é sempre 
prudente, em estudos sobre testes diagnósticos, contabilizar um resultado verdadeiramente negativo ou positivo, mediante a pré-definição de critérios para a aplicação de um teste, mesmo que este seja padrão-ouro ${ }^{19}$.

Dessa forma, preocupados com o viés de verificação, busca-se avaliar o erro intrínseco intraobservador no cálculo do ângulo de Cobb em sujeitos escolióticos e analisar a correlação interobservadores no cálculo do ângulo de Cobb em sujeitos escolióticos.

\section{MÉTODO}

Participaram do estudo vinte e cinco sujeitos, selecionados aleatoriamente, com escoliose estrutural idiopática. Foram excluídos do estudo 8 indivíduos por terem mais de 21 anos de idade, ou por relatarem cirurgia prévia da coluna vertebral, fratura, gestação ou neoplasia. Os 17 indivíduos (7 meninos e 10 meninas) que foram selecionados, conforme análise dos prontuários, estavam na faixa etária entre 9 e 20 anos e apresentavam escoliose idiopática de grau leve, na maioria dos casos (ângulo de Cobb com variações entre 5 e 21 graus). A média de idade de $14( \pm 4)$ anos, de altura de $169,5 \mathrm{~cm}( \pm 0,09)$ e de massa $56,5 \mathrm{~kg}$ $( \pm 9,12)$. Todos os pacientes estavam realizando radiografias para acompanhamento de suas escolioses no Hospital Universitário de Santa Catarina - HU. Essas radiografias foram feitas mediante controle do erro extrínseco, isto é, na mesma hora, posição e distância, pelo mesmo profissional e, ainda, no mesmo equipamento de raio- $\mathrm{X}^{20}$. A pesquisa teve a aprovação do Comitê de ética e pesquisa em Seres Humanos da Universidade do Estado de Santa Catarina UDESC (protocolo n ${ }^{\circ}$ 5/99).

Previamente à análise das 34 radiografias dos sujeitos (incidências ântero-posterior e perfil), os três observadores, receberam uma explanação teórica e um informativo, com instrução da técnica de análise do cálculo do ân- gulo de Cobb. Todos os observadores possuíam mais de cinco anos de experiência em ortopedia-traumatologia, sendo um deles cirurgião ortopedista e dois fisioterapeutas com o título de mestre. O material foi elaborado por um ortopedista com mais de quinze anos de experiência e seguiu as orientações da literatura ${ }^{21}$.

O treinamento prático no raio-x foi encerrado quando os observadores estavam inteiramente familiarizados com a seleção na marcação das vértebras e com a interpretação angular. Esse raio-x intitulado exame-teste foi eliminado da pesquisa. Os observadores utilizaram-se do mesmo lápis, da mesma régua (50 $\mathrm{cm})^{8,13,22}$ e fizeram a leitura, igualmente, com o mesmo goniômetro de dupla haste (Kroman $\left.{ }^{\circledR}\right)$.

Para verificação da concordância as radiografias foram avaliadas, independentemente e em condições idênticas, por todos os avaliadores. Os valores interobservadores foram oriundos das avaliações dos três observadores. Para que cada observador não sofresse influência na seleção das vértebras, após a leitura e a marcação em planilha própria das vértebras selecionadas e, consequentemente, do valor do ângulo de Cobb, o pesquisador apagava todas as marcações com algodão embebido em álcool, eliminando assim qualquer interferência para o próximo observador. Os profissionais não receberam informações sobre o quadro clínico dos pacientes avaliados.

A curvatura escoliótica é caracterizada com a identificação das vértebras terminais, em que a última vértebra é assinalada em cada extremidade que está inclinada para a concavidade da curva. Essas vértebras são marcadas com duas linhas horizontais, uma na placa terminal inferior e outra na placa terminal superior. Na sequência, desenham-se linhas perpendiculares a elas, e o ângulo formado pelo encontro das duas linhas é medido pelo goniômetro. Em curvas maiores, o ângulo formado pelas linhas terminais é medido diretamente ${ }^{5,12}$.

Foi sorteado apenas um dos avaliadores, para mensurar a concordância intraobserva- 
dor ${ }^{11}$. Com o intuito de avaliar o erro intrínseco no ângulo de Cobb, utilizou-se da equação proposta por Morrissy et al ${ }^{13}$ :

$$
\frac{\text { " } \Sigma\left[\left(\mathrm{U}_{1}-\mathrm{U}_{2}\right)^{2}+\left(\mathrm{L}_{1}-\mathrm{L}_{2}\right)^{2}\right]^{1 / 2}}{\text { Número total de curvas encontradas }}
$$

onde:

$\mathrm{U}_{1}, \mathrm{U}_{2}$ ("upper"), $\mathrm{L}_{1}$ e $\mathrm{L}_{2}$ ("lower") foram as $1^{\text {as }} \mathrm{e} 2^{\text {as }}$ escolhas (cefálica e caudal) das vértebras selecionadas para o cálculo do ângulo de Cobb”. Para a análise, foi utilizado a média dos erros intrínsecos dos três níveis da coluna vertebral.

O tempo de intervalo entre a $1^{\mathrm{a}}$ e a $2^{\mathrm{a}}$ avaliação foi de 45 dias e da $2^{\mathrm{a}}$ avaliação para a $3^{\text {a }}$ avaliação decorreram 3 dias $^{23}$.

Por meio de uma análise descritiva dos dados, a concordância interobservadores foi realizada mediante o coeficiente de correlação do momento do produto de Pearson (r), juntamente com os valores do Teste ANOVA One Way e o Teste de Newman-Keuls. Na análise intraobservador foi utilizado, igualmente, o coeficiente de correlação do momento do produto Pearson (r) e o Teste " $\mathrm{t}$ " 24 . Os procedimentos estatísticos foram realizados utilizando-se do programa Statistica ${ }^{\circledR}$ versão 6.0. O nível de significância para esta pesquisa foi de $5 \%$ $(\mathrm{p} \leq 0,05)$.

\section{RESULTADOS}

Os dados referentes ao número de curvas encontradas pelos diferentes avaliadores durante a avaliação da imagem radiológica são apresentados na tabela 1 .

Tabela 1: Número de curvas escolióticas encontradas pelos diferentes avaliadores, na avaliação radiológica (incidência ântero-posterior), segundo ângulo de Cobb.

\begin{tabular}{ccccc}
\hline & \multicolumn{2}{c}{ Avaliadores do ângulo de Cobb no Raio-x } & Média \\
Curvas: & Avaliador 1 & Avaliador 2 & Avaliador 3 & - \\
\hline Torácica & 12 & 12 & 15 & 13 \\
Lombar & 8 & 9 & 8 & 8 \\
Tóraco-lombar & 8 & 5 & 6 & 6 \\
Total de curvas & 28 & 26 & 29 & 27 \\
\hline
\end{tabular}

Fonte: Clínica escola da UDESC em Florianópolis/SC.

\section{Cálculo da variabilidade intra-observador}

As diferenças angulares, entre a $2^{\mathrm{a}}$ e a $3^{\mathrm{a}}$ avaliação intraobservador estão demonstradas na tabela 2. Cerca de $87 \%$ das mensurações evidenciaram diferença de até três graus entre os ângulos de Cobb.

Quanto ao cálculo do índice de erro, este sofreu influencia da variabilidade na escolha das vértebras. Os valores encontrados na escolha das vértebras foram de 0,26 para o nível torácico, 0,20 para o tóraco-lombar e 0,07 para o nível lombar. Assim, a média dos erros intrínsecos foi de 0,17. Estes valores foram obtidos mediante a equação proposta por Morrissy
Tabela 2: Dados referentes à diferença intraobservador entre os ângulos de Cobb encontrados na $2^{\mathrm{a}}$ para a $3^{\mathrm{a}}$ avaliação.

\begin{tabular}{lrr}
\hline Graus & $\mathbf{F a}^{\mathbf{2}}$ & $\mathbf{F a c}^{\mathbf{3}}$ \\
\hline Nula & 6 & 6 \\
1 grau & 7 & 13 \\
1,5 graus & 1 & 14 \\
2 graus & 7 & 21 \\
3 graus & 7 & 28 \\
4 graus & 1 & 29 \\
5 graus & 2 & 31 \\
7 graus & 1 & 32 \\
Total & 32 & 174 \\
\hline
\end{tabular}

1 - Diferença em graus da $2^{\text {a }}$ para a $3^{\mathrm{a}}$ avaliação, 2 - Frequência absoluta, 3 - Frequência absoluta acumulada.

Fonte: Clínica escola da UDESC em Florianópolis/SC. 
et $\mathrm{al}^{13}$. Este índice é um método que expressa a variabilidade na escolha da vértebra final (caudal) em duas diferentes ocasiões. Quanto maior o índice de erro, mais variável é a escolha da vértebra caudal, entre medidas sucessivas, na mesma radiografia. Menor índice indica uma escolha mais consistente da vértebra para replicar as medidas dos ângulos de Cobb.

\section{Concordância intraobservador para o ângulo de Cobb}

Para o nível torácico dos sujeitos escolióticos, não houve diferença significativa no Teste " $\mathrm{t}$ " $(\mathrm{p}=0,18)$. Esses valores estão demonstrados na tabela 3 . Além disso, houve relação significativa excelente ${ }^{8}(r=0,80$; $\left.r^{2}=0,64 ; p=0,001\right)$ nas mensurações do ân- gulo de Cobb intraobservador. Mior et al. ${ }^{8}$ considera que uma correlação abaixo de 0,55 é insatisfatória, entre 0,55 e 0,64 é fraca, entre 0,65 e 0,76 é boa e, acima de 0,77 é excelente, para alfa de 0,005 e $95 \%$ de intervalo de confiança.

Para o nível tóraco-lombar desses sujeitos não houve, igualmente, diferença estatisticamente significativa ( $\mathrm{p}=0,86)$. Estes valores estão demonstrados na tabela 4 . O ângulo de Cobb intra-observador para o nível tóracolombar apresentou correlação, igualmente, excelente $^{8}\left(r=0,80 ; r^{2}=0,63 ; p=0,031\right)$.

O nível lombar, igualmente, não apresentou diferença significativa no Teste " $\mathrm{t}$ " ( $\mathrm{p}$ = $0,69)$. Estes valores estão demonstrados na tabela 5 . Não houve correlação significativa ${ }^{8}$

Tabela 3: Valores do ângulo de Cobb, para o Teste “t” intra-avaliador, no nível torácico em sujeitos escolióticos.

\begin{tabular}{cccccc}
\hline Avaliação & Média & Desvio padrão & $\mathbf{N}$ & $\mathbf{~}$ & $\mathbf{p}$ \\
\hline $2^{\mathrm{a}}$ & 5,31 & 3,77 & 16 & $-1,39$ & 0,18 \\
$3^{\mathrm{a}}$ & 6,25 & 4,53 & 16 & - & - \\
Média & 5,78 & 4,15 & - & - & - \\
\hline
\end{tabular}

Fonte: Clínica escola da UDESC em Florianópolis/SC.

Tabela 4: Valores do ângulo de Cobb, para o Teste " $t$ ” intra-avaliador, no nível tóraco-lombar em sujeitos escolióticos.

\begin{tabular}{cccccc}
\hline Avaliação & Média & Desvio padrão & $\mathbf{~ N}$ & $\mathbf{t}$ & $\mathbf{p}$ \\
\hline $2^{\mathrm{a}}$ & 7,14 & 3,13 & 7 & $-0,18$ & 0,86 \\
' $^{\mathrm{a}}$ & 7,28 & 3,45 & - & - & - \\
Média & 7,21 & 3,29 & - & - & - \\
\hline
\end{tabular}

Fonte: Clínica escola da UDESC em Florianópolis/SC.

intra-observador $\left(\mathrm{r}=0,62 ; \mathrm{r}^{2}=0,39 ; \mathrm{p}=0,07\right)$ para esse nível da coluna vertebral.

\section{Concordância interobservador para o ângulo de Cobb}

Os valores do coeficiente de correlação do momento do produto Pearson (r), para o ângulo de Cobb interobservador, nos diferen- tes níveis da coluna vertebral são apresentados na tabela 6.

Houve relação, estatisticamente significativa, no nível torácico $(\mathrm{p} \leq 0,05)$ entre as medidas realizadas pelos diferentes observadores. Os coeficientes de correlação entre os observadores 1 , 2 e 3 oscilaram de bom $(0,65$ e 0,76$)$ a excelente (acima de 0,77 ), sendo mais evi- 
Tabela 5: Valores do ângulo de Cobb, para o Teste "t" intra-avaliador, no nível lombar em sujeitos escolióticos.

\begin{tabular}{cccccc}
\hline Avaliação & Média & Desvio padrão & $\mathbf{~ N}$ & $\mathbf{t}$ & p \\
\hline $2^{\text {a }}$ & 5,72 & 3,09 & 9 & 0,41 & 0,69 \\
$3^{\text {a }}$ & 5,33 & 3.42 & 9 & - & - \\
Média & 5,52 & 3,09 & - & - & - \\
\hline
\end{tabular}

Fonte: Clínica escola da UDESC em Florianópolis/SC.

Tabela 6: Valores dos coeficientes de associação (r) e determinação $\left(\mathrm{r}^{2}\right)$ interobservadores, em indivíduos escolióticos, para o nível torácico (T), tóraco-lombar (TL) e lombar (L). Avaliação radiológica, segundo ângulo de Cobb.

\begin{tabular}{ccccc}
\hline Observadores & Número de curvas & $\mathbf{r}$ & $\mathbf{r}^{2}$ & $\mathbf{p}$ \\
\hline 1 e 2(T) & 9 & 0,82 & 0,68 & 0,006 \\
1 e 3(T) & 11 & 0,85 & 0,74 & 0,001 \\
2 e 3(T) & 12 & 0,69 & 0,49 & 0,012 \\
1 e 2(TL) & $2 *$ & $1,00^{*}$ & $-*$ & $-*$ \\
1 e 3(TL) & 4 & 0,76 & 0,58 & 0,23 \\
2 e 3(TL) & 3 & 0,98 & 0,78 & 0,099 \\
1 e 2(L) & 6 & 0,007 & $0 * *$ & 0,98 \\
1 e 3(L) & 5 & 0,18 & 0,03 & 0,76 \\
2 e 3(L) & 5 & 0,85 & 0,72 & 0,06 \\
\hline
\end{tabular}

* número reduzido de curvas (apenas duas por avaliador), o que poderá justificar os valores em $\mathrm{r}$, $\mathrm{r}^{2}$ e $\mathrm{p}^{* *}$. Este valor indica que um avaliador não possui qualquer inferência na medida do outro, ou seja, similaridade nas suas medidas.

Fonte: Clínica escola da UDESC em Florianópolis/SC.

dente entre os observadores 1 e 3 . O nível tóraco-lombar, de maneira geral, evidenciou diferenças entre os observadores, à exceção da perfeita correlação entre os observadores 1 e 2. Tanto o $2^{\circ}$ quanto o $3^{\circ}$ observador apresentaram coeficiente de associação (r) igual a 0,98 , mas em um número reduzido de curvas escolióticas. No nível lombar, os observadores não apresentaram correlação significativa entre si.

A ANOVA one way das médias do ângulo de Cobb interobservadores (observador 1 ; observador 2 e observador 3) da coluna torácica em sujeitos escolióticos, não demonstrou diferenças estatisticamente significativas entre elas, anova $=0,46$ e $p=0,63$. A média dos valores referente à coluna tóraco-lombar interobservador (observador 1; observador 2 e observador 3), não evidenciou, novamente, diferenças significativas entre elas, anova $=0,39$ e p = 0,67. A média dos valores referente à coluna lombar interobservador (observador 1; observador 2 e observador 3) não mostrou diferença significativa, anova $=1,43$ e $\mathrm{p}=0,26$. Consequuentemente, não existiu diferença entre o grupo de observadores na análise do ângulo de Cobb em escolióticos ( $\mathrm{p} \geq 0,05)$.

\section{DISCUSSÃO}

É prudente lembrar que não houve, por parte dos pesquisadores, uma pré-seleção das vértebras da coluna vertebral, que caracterizavam a escoliose. Sendo assim, quando o observador selecionava, por exemplo, T12 ou L1, automaticamente, determinava o nível da escoliose, o que justifica em uma análise final, a diferença no número total de curvas encontradas por cada observador. A pré-seleção das 
vértebras que compõem a curvatura minimiza o erro entre as avaliações intra e interobservadores ${ }^{13}$.

Grande parte das mensurações apresentou diferenças de até três graus intraobservador. Lonstein e Carlson ${ }^{25}$ usam cinco graus de diferença entre os ângulos de Cobb, entre duas sucessivas radiografias, como critério de progressão das curvaturas escolióticas. Sabe-se que as maiores deformidades rotacionais afetam a coluna torácica, onde menos de cinco graus de curvatura pode ser considerado "falso positivo" 26 . Contudo, o erro intraobservador não interfere em situações de vida real, em que há necessidade de classificar a severidade das curvaturas escolióticas.

Entende-se que o número total de curvas encontradas na região lombar influenciou significativamente nos valores apontados neste estudo, apesar do Teste "t" evidenciar a não existência de diferenças entre as médias, em função do nível de significância colocado pelos pesquisadores.

Gross, Gross e Kuschner ${ }^{16}$ orientaram uma pesquisa em que três observadores durante cinco vezes, avaliaram o erro de mensuração de curvas entre $4,5^{\circ}$ e $106,5^{\circ}$. O erro intraobservador variou entre \pm 2 a \pm 4 graus. Já a ausência dos valores intra-observador foi apontada como uma limitação em estudos com metodologia similar ${ }^{15,17}$.

Nossos resultados evidenciaram uma média para o nível torácico de $5,78^{\circ}$, para o nível tóraco-lombar de $7,21^{\circ}$ e para o nível lombar de $5,52^{\circ}$. A média total apontou para uma diferença de $6,17^{\circ}$ entre as sucessivas mensurações do ângulo de Cobb. Morrissy et al ${ }^{13}$ encontraram cerca de $4,9^{\circ}$ de diferença intraobservador em pacientes com escoliose, quando a vértebra era selecionada pelo próprio observador e com o mesmo instrumento de mensuração. Estudos prévios $^{13,16}$ comprovaram a diferença interobservador entre $6^{\circ}-7^{\circ} \mathrm{em} 95 \%$ da amostra estudada.
A reprodutibilidade do ângulo de Cobb em escolióticos idiopáticos (juvenil e adolescente) foi, igualmente, medida mais recentemente por Modi et $a l^{18}$. Neste estudo, não houve diferenças significativas em todas as mensurações para ambos os observadores na escoliose idiopática juvenil (anova com variação de 0,706 a 0,815 e p $=0,756$ ) e na escoliose idiopática do adolescente (anova com variação de 0,795 a 0,929 e $\mathrm{p}=0,871$ ). A reprodutibilidade intraobservador foi menor na escoliose idiopática juvenil $(\mathrm{r}=0,60)$ ao ser comparado com a escoliose idiopática do adolescente $(r=0.96)$, apresentando, ainda, resultados similares interobservador, isto é, escoliose idiopática juvenil $(r=0.54)$ e escoliose idiopática do adolescente $(\mathrm{r}=0.96)$.

A análise dos resultados interobservador, nos três níveis da coluna vertebral, apontou para a existência de correlações entre os diferentes observadores. Especificamente, os observadores no nível torácico apresentaram excelentes associações e concordâncias entre si, diferentemente da coluna lombar, que é, geralmente, parte de uma dupla curvatura, particularmente visível em análises dos ápices nos planos frontais ${ }^{27}$. Por outro lado, o tradicional uso dos filmes planos reduz para duas dimensões uma deformidade que ocorre nos três planos ${ }^{28}$.

Similar a estudo prévio Nissinen et al ${ }^{1}$, tanto a coluna tóraco-lombar quanto a lombar, não apresentaram valores concordantes entre os diferentes observadores, merecendo uma atenção especial, uma vez que, na maioria dos casos, as escolioses dominantes, encontram-se no nível torácico, mas com repercussões clínicas na região lombar. Dang et al ${ }^{22}$ descrevem que o erro nas avaliações de tais níveis pode ser, ainda, atribuído à falta de claridade em radiografias e a não seleção e definição da última vértebra (lombar ou sacral).

Considerando tais informações, supõese necessária a criação de um sistema de vigilância sobre diagnoses da coluna vertebral, 
orientado, principalmente, ao controle do erro de mensuração. Complementar a essas informações Ferreira et al ${ }^{29}$ encontraram, mediante a utilização de testes não-invasivos, elevados índices de escoliose na população de escolares brasileiros. Além do mais, deixam clara a necessidade da confirmação da deformidade, via exame radiográfico.

Razões de ordem prática como estas, ajudam-nos a elaborar programas de rastreamento da escoliose, bem como, estimular a construção de estratégias de tratamento precoce, tendo em vista os subdiagnósticos, as intervenções cirúrgicas tardias, o que, em longo prazo, poderá minimizar a sobrecarga dos cofres públicos nacionais.

Não houve diferenças significativas de julgamento na condição intra e interobservadores. A magnitude dos coeficientes de cor-

\section{REFERÊNCIAS}

1. Nissinen MJ, Heliovaara MM, Seitsamo JT, Kononen MH, Hurmerinta KA, Poussa MS. Development of trunk asymmetry in a cohort of children ages 11 to 22 years. Spine. 2000; 25(5): 570-574.

2. Hawes MC. The use of exercises in the treatment of scoliosis: An evidence-based critical review of the literature. Pediatr Rehabil. 2003; 6(3-4): 171-182.

3. Tosato JP, Caria PHF. Avaliação da atividade muscular na escoliose. Rev Bras Crescimento Desenvol Hum. 2009; 19(1): 98-102.

4. Forlin E, Vieira LFT. Avaliação da acurácia da determinação da idade óssea e de sua aplicação na projeção da discrepância final do comprimento dos membros inferiores. Rev Bras Ortop. 1994; 29: 597-600.

5. Bradford DS, Lostein JE, Moe JH, Ogilvie JW, Winter RB. Escoliose e outras deformidades da coluna "O livro de Moe". São Paulo: Santos; 1994. relação variou de excelente para o nível torácico, na condição intraobservador e de bom/excelente nas avaliações interobservador; excelente para o nível tóraco-lombar na condição intraobservador e de boa/excelente na categoria interobservador e nula na coluna lombar em ambas as condições.

\section{AGRADECIMENTOS}

Agradecemos a colaboração dos profissionais envolvidos nas análises dos ângulos de Cobb, o auxílio técnico dos radiologistas e o apoio dos ortopedistas e traumatologistas do Hospital Universitário Ernani Polydoro de São Thiago - UFSC/SC. Reconhecimento especial ao professor Dr. Francisco Rosa Neto pelo delineamento da pesquisa.

6. Kleinerman RA. Cancer risks following diagnostic and therapeutic radiation exposure in children. Pediatr Radiol. 2006; 36: 121-125.

7. Doody M, Lonstein JE, Stovall M, Hacker DG, Luckyanov N, Land CE. Breast cancer mortality after diagnostic radiography: findings from the U.S. Scoliosis Cohort Study. Spine. 2000; 25(16): 2052-2063.

8. Mior SA, Kopansky-Giles DR, Crowther E, Wright JG.: A comparison of radiographic and eletrogoniometric angles in adolescent idiopathic scoliosis. Spine. 1996; 21(13): 1549-1555.

9. Dao TV, Labelle H, LE BLANC R. Variabilité intra-observateur de la mesure de la posture à láide dún numérisateur tridimensionnel. Ann Chir. 1997; 51(8): 848-853.

10. Ovadia D, Bar-On E, Fragnière B, Rigo M, Dickman D, Leitner J, et al. Radiationfree quantitative assessment of scoliosis: a multi center prospective study. Eur Spine J. 2007; 16(1): 97- 105. 
11. Pruijs JEH, Keessen W, Van der Meer R, van Wieringen JC, Hageman MAPE. School screening for Scoliosis: Methodologic Considerations- Part 1: external measurements. Spine. 1992; 17: 431-435.

12. Carman DL, Browne RH, Birch JG. Measurement of scoliosis and kyphosis radiographs. Intraobserver and interobserver variation. J Bone Joint Surg Am. 1990; 72(3):328-33.

13. Morrissy RT, Goldsmith GS, Hall EC, Kehl D, Cowie GH. Measurement of the Cobb angle on radiographs of patients who have scoliosis - Evaluation of intrinsic error. $\mathrm{J}$ Bone Joint Surg. 1990; 72-A: 320-327.

14. Shea KG, Stevens PM, Nelson M, Smith JT, Masters K S, Yandow SA. Comparison of manual versus computer-assisted radiographic measurement. Intraobserver measurement variability for Cobb angles. Spine. 1998; 23: 551-555.

15. Sevastikoglou JA, Bergquist E. Evaluation of the reliability of radiological methods for registration of scoliosis. Acta Orthop Scand. 1969; 40(5): 608-613.

16. Gross C, Gross M, Kuschner S. Error analysis of scoliosis curvature measurement: Bull Hosp Jt Dis Orthop Inst. 1983; 43(2): 171-177.

17. Beekmann CE, Hall V. Variability of scoliosis measurement from spinal roentgenograms. Phys Ther. 1979; 59: 764-765.

18. Modi HN, Chen T, Suh SW, Mehta S, Srinivasalu S, Yang J, et al. Observer reliability between juvenile and adolescent idiopathic scoliosis in measurement of stable Cobb's and angle. Eur Spine J. 2009; 18: 52-58.

19. Hulley SB, Cummings SR, Browner WS, Grady DG, Newman TB. Delineando a pesquisa clínica - Uma abordagem epidemiológica. Porto Alegre: Artmed; 2008.
20. Leroux MA, Zabjek K, Simard G, Badeaux J, Coillard C, Rivard CH. A noninvasive anthropometric technique for measurement kyphosis and lordosis: an application for idiopathic scoliosis. Spine. 2000; 25 (13): 1689-94.

21. Vedantam R, Lenke LG, Kenney JA, Bridwell $\mathrm{KH}$. Comparison of standing sagital spinal alignment in asymptomatic adolescents and adults. Spine. 1998; 23(2): 211-5.

22. Dang NR, Moreau MJ, Hill DL, Mahood JK, Raso J. Intra-observer Reproducibility and Interobserver Reliability of the Radiographic Parameters in the Spinal Deformity Study Group's AIS Radiographic Measurement Manual. Spine. 2005; 30(9):1064-1069.

23. Delisa JA. Tratado de medicina de reabilitação. São Paulo: Manole; 2002.

24. Bisquera R, Sarrriera JC, Martinez F. Introdução a estatística - Enfoque informático com o pacote estatístico SPSS. Porto Alegre: Artmed; 2004.

25. Lonstein JE, Carlson JM. The prediction of curve progression in untreated idiopathic scoliosis during growth. J Bone Joint Surg Am. 1984; 66:1061-1071.

26. Bunnell WP, Delaware W. An objective criterion for scoliosis screening. J Bone Joint Surg. 1984; 66-A: 1381-1387.

27. Gram MC, Hasan Z. The spinal curve in standing and sitting posture in children with idiopathic scoliosis. Spine. 1999; 24(2): 169-177.

28. Birchall D, Hughes D, Hindle J, Robinson L, Williamson JB. Measurement of vertebral rotation in adolescent idiopathic scoliosis using three-dimensional magnetic resonance imaging. Spine.1997; 22 (20): 2403-2407.

29. Ferreira DMA, Suguikawa, TR, Pachioni, CAS, Fregonesi, CEPT, Camargo, MR. Rastreamento escolar da escoliose: medida para o diagnóstico precoce. Rev Bras Crescimento Desenvolv Hum. 2009; 19(3): 357-368.

Recebido em: 25/out./10 Modificado em: 02/nov./10 Aceito em: 08/dez./10 